\title{
Assessment of Ifosfamide Pharmacokinetics, Toxicity, and Relation to CYP3A4 Activity as Measured by the Erythromycin Breath Test in Patients With Sarcoma
}

\author{
Rashmi Chugh, MD ${ }^{1}$ \\ Thomas Wagner, $\mathrm{MD}, \mathrm{PhD}^{2}$ \\ Kent A. Griffith, MPH, Ms ${ }^{3}$ \\ Jeremy M.G. Taylor, PhD ${ }^{3}$ \\ Dafydd G. Thomas, MD, PhD ${ }^{1}$ \\ Francis $P$. Worden, MD ${ }^{1}$ \\ Kirsten M. Leu, $\mathrm{MD}^{4}$ \\ Mark M. Zalupski, MD ${ }^{1}$ \\ Laurence H. Baker, Do ${ }^{1}$
}

\footnotetext{
${ }^{1}$ Department of Internal Medicine, Division of Hematology/Oncology, University of Michigan Medical School, Ann Arbor, Michigan.

${ }^{2}$ Division of Hematology/Oncology, University of Lubeck, Lubeck Germany.

${ }^{3}$ Department of Biostatistics, University of Michigan Comprehensive Cancer Center, Ann Arbor, Michigan.
}

${ }^{4}$ Oncology Hematology West, PC, Omaha, Nebraska.
Supported by Amgen, Inc.

Dr. Wagner has acted as a consultant for Baxter Oncology.

Dr. Baker has received grant support from Abbott, Aventis (Sanofi), Lilly, the National Institutes of Health, the Robert Urich Foundation, the Southwest Oncology Group, the Walther Foundation, and the Hyatt Corporation and is a member of the Advisory

BACKGROUND. Ifosfamide is a chemotherapeutic agent that requires cytochrome P450 3A (CYP3A) for bioactivation and metabolism. To the authors' knowledge, the correlation between dose, pharmacokinetics, CYP3A, and toxicity has not been fully evaluated. A randomized Phase II trial was performed on 22 soft tissue sarcoma patients treated with doxorubicin $\left(60 \mathrm{mg} / \mathrm{m}^{2} /\right.$ cycle $)$ and either highdose ifosfamide $\left(12 \mathrm{~g} / \mathrm{m}^{2} /\right.$ cycle $)$ or standard-dose ifosfamide $\left(6 \mathrm{~g} / \mathrm{m}^{2} /\right.$ cycle $)$. The pharmacokinetics of ifosfamide and CYP3A measurements observed are reported. METHODS. Pharmacokinetic parameters for ifosfamide, 2-dichloroethylifosfamide (2-DCE), and 3-dichloroethylifosfamide (3-DCE) were collected after the first ifosfamide infusion in 13 patients. Bayesian designed limited pharmacokinetic data were collected from an additional 41 patients. The erythromycin breath test (ERMBT) was performed on 81 patients as an in vivo phenotypic assessment of CYP3A activity.

RESULTS. Fourteen-hour (peak) plasma levels of ifosfamide, 2-DCE, and 3-DCE were found to correlate strongly with the respective area under the curve (AUC) $0-24$ values $(r=0.97,0.94$, and $0.95 ; P<.0001)$. Patients who experienced a grade 3-4 absolute neutrophil count (ANC), platelet, or creatinine toxicity (using the National Cancer Institute Common Toxicity Criteria [version 2]) were found to have statistically significantly higher median 14-hour plasma levels of ifosfamide, 2-DCE, and 3-DCE compared with patients with grade 0-2 toxicity. ERMBT was not found to correlate with pharmacokinetic parameters of ifosfamide and metabolites or toxicity.

CONCLUSIONS. The 14-hour plasma level of ifosfamide, 2-DCE, and 3-DCE is a simple and appropriate substitute for describing the AUC of ifosfamide after 1 day of a 1-hour to 2-hour infusion of drug. Fourteen-hour plasma levels of ifosfamide and metabolites are useful predictors of neutropenia, thrombocytopenia, and creatinine toxicity. ERMBT was not found to accurately correlate with ifosfamide pharmacokinetics or clinical toxicity. Cancer 2007;109:2315-22. () 2007 American Cancer Society.

KEYWORDS: ifosfamide, cytochrome P450 3A, erythromycin breath test, sarcoma, pharmacokinetics.

Boards of Ascenta, the Hope Foundation (Vice President), the NCCN Guidelines Committee, Chairman, Southwest Oncology Group, and SARC (Executive Director and Board Member).

Presented at the 38th Annual Meeting of the American Society of Clinical Oncology, Orlando, Florida, May 19, 2002; the 39th Annual Meeting of the American Society of Clinical Oncology, Chicago, Illinois, June 2, 2003; and the 41st
Annual Meeting of the American Society of Clinical Oncology, Orlando, Florida, May 13-17, 2005.

Address for reprints: Laurence H. Baker, D0, 24 Frank Lloyd Wright Drive, PO Box 483, Ann Arbor, Ml 48106; Fax: (734) 998-7118; E-mail: baker|@umich.edu, bakerl@umich.edu

Received September 19, 2006; revision received January 23, 2007; accepted January 24, 2007. 
I fosfamide is a cancer chemotherapeutic drug that belongs to the oxazaphosphorine class of alkylating agents. Significant clinical toxicities involve the central nervous system and the genitourinary system, in addition to myelosuppression, nausea, emesis, alopecia, and elevated liver enzymes. ${ }^{1-3}$ Ifosfamide has complex pharmacokinetics (Fig. 1), which have been extensively reviewed. The cytochrome P450 3A (CYP3A) family, mainly CYP3A4 and CYP3A5, are the major enzymes involved in bioactivation of this prodrug. ${ }^{4,5}$ Cytochrome $2 \mathrm{~B} 6$ has also been implicated to a minor degree. ${ }^{6,7}$ This process involves 4-hydroxylation and ultimately yields phosphoramide mustard and acrolein. ${ }^{8,9}$ Phosphoramide mustard is the therapeutically active metabolite that possesses DNA alkylating activity. ${ }^{9,10}$ Acrolein is an inactive agent that is responsible for bladder toxicity.

A second metabolic pathway of ifosfamide involves detoxification of the drug by side-chain $\mathrm{N}$ dechlorethylation (Fig. 1). CYP3A is the main enzyme responsible for the formation of therapeutically inactive metabolites 2-dechloroethylifosfamide (2-DCE) and 3-dechlorethylifosfamide (3-DCE) ${ }^{5,6}$ The creation of 2-DCE and 3-DCE yields equal amounts of chloroacetaldehyde, the metabolite responsible for neurotoxicity from ifosfamide. , $11,12^{\text {Chloroacetaldehyde is }}$ rapidly degraded, ${ }^{13}$ and 2-DCE and 3-DCE measurements are often preferred assessments of this pathway.

Given its complex pharmacokinetics and the potential for severe toxicities, we wished to better understand the relation between metabolite levels and ifosfamide toxicity and to explore the use of a predictive test of hepatic CYP3A activity when using ifosfamide. The erythromycin breath test (ERMBT) is performed by intravenous injection of a trace dose of radiolabeled erythromycin. CYP3A is the major enzyme catalyzing the subsequent $\mathrm{N}$-demethylation of erythromycin and conversion to carbon dioxide. ${ }^{14}$ Exhaled radiolabeled carbon dioxide is then measured to quantify this reaction. ${ }^{15}$

This test has been validated as a measure of CYP3A activity. We have previously shown that the ERMBT correlates with lower CYP3A activity levels and clinical toxicity ${ }^{16}$ in patients receiving docetaxel, a drug metabolized by the CYP3A system. To our knowledge, there has been no prior report of using predictive tests to assay CYP3A activity to assess the pharmacokinetics and toxicity of ifosfamide. ${ }^{2}$

Recently, we reported on a Phase II evaluation of high-dose ifosfamide $\left(12 \mathrm{~g} / \mathrm{m}^{2} /\right.$ cycle $)$ versus standard dose ifosfamide $\left(6 \mathrm{~g} / \mathrm{m}^{2} /\right.$ cycle $)$ in conjunction with doxorubicin (60 $\mathrm{mg} / \mathrm{m}^{2} /$ cycle) in patients with soft tissue sarcoma. ${ }^{17}$ To better understand and predict

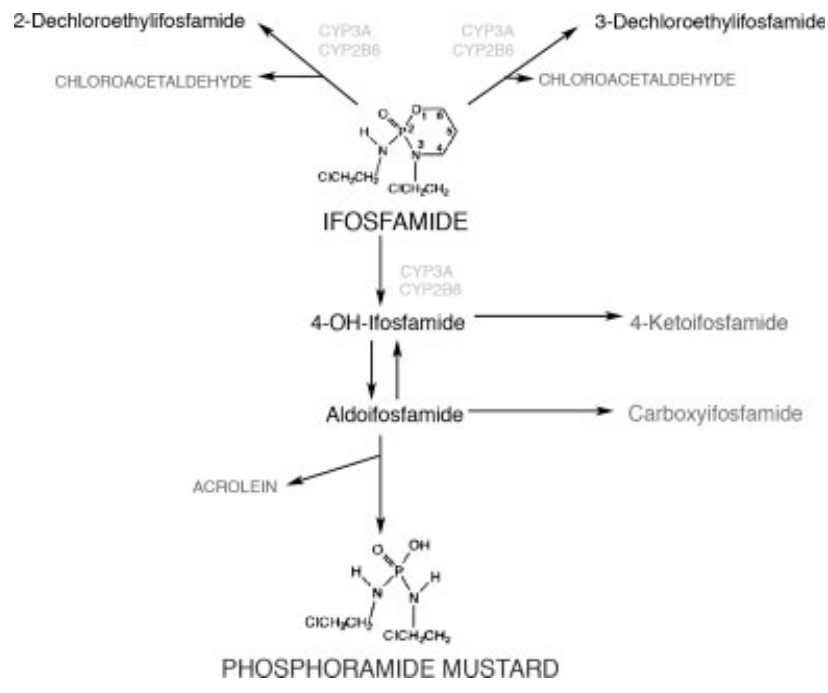

FIGURE 1. Activation and metabolism of ifosfamide.

the pharmacokinetics and toxicity of these treatments, we performed serum and urine measurements of ifosfamide and metabolites (2-DCE, 3-DCE) and ERMBT to assess CYP3A activity. Herein, we examine the correlation between dose, drug and metabolite levels, ERMBT, and toxicities.

\section{MATERIALS AND METHODS \\ Trial Design}

Eligible patients were enrolled in a single-institution randomized Phase II trial. Eligibility criteria included patients with documented soft tissue sarcoma, American Joint Committee on Cancer (AJCC) Stage IIIB disease (grade $\mathrm{G} 3,>5 \mathrm{~cm}$ in greatest dimension); AJCC Stage IIB disease (grade G2, $>5 \mathrm{~cm}$ in greatest dimension and deep to initial fascia); or patients with measurable, metastatic, high-grade $(\geq \mathrm{G} 2$ lesions) disease who were previously untreated with chemotherapy. Patients who had undergone primary surgical resection received adjuvant chemotherapy, whereas patients with unresected primary disease received neoadjuvant chemotherapy. Patients with metastases received palliative treatment. Participants were required to be at least 16 years old with a Zubrod performance status of $\leq 2$. Further eligibility requirements included an absolute neutrophil count (ANC) of $\geq 1500$ cells $/ \mathrm{mm}^{3}$, a platelet count of $\geq 100,000$ cells $/ \mathrm{mm}^{3}$, total bilirubin $\leq 1.5$ times the upper limit of normal (ULN), and a serum creatinine $\leq 1.2$ times the ULN. Patients were required to have 2 functioning kidneys and an estimated creatinine clearance of $\geq 50 \mathrm{cc} / \mathrm{min}$. The study was approved by the local Institutional Review Board and written informed consent was obtained from each patient. 
Patients were randomized to receive either highdose ifosfamide $\left(3.0 \mathrm{~g} / \mathrm{m}^{2} /\right.$ day given over $1-2$ hours on Days 1-4) and mesna (1800 $\mathrm{mg} / \mathrm{m}^{2} /$ day on Days $1-4)$ or standard dose ifosfamide $\left(1.5 \mathrm{~g} / \mathrm{m}^{2} /\right.$ day given over 1-2 hours on Days 1-4) and mesna (1800 mg/ $\mathrm{m}^{2} /$ day on Days 1-4). All patients received doxorubicin $\left(60 \mathrm{mg} / \mathrm{m}^{2}\right.$ given as a 72 -hour continuous infusion starting Day 1) and filgrastim (5 $\mathrm{mcg} / \mathrm{kg} / \mathrm{dose}$ ) until the ANC was $>1500$ cells $/ \mathrm{mm}^{3}$. Details of the clinical design and primary clinical results have been previously published. ${ }^{17}$

\section{Laboratory Monitoring}

Before enrollment, patients had a history and physical examination. Pretherapy laboratory studies included a complete blood cell count with differential and platelet counts (CDP) and serum chemistries. Chemistries were monitored weekly and before each cycle. A CDP was obtained on Days 1, 8, 10, 12, 15,17 , and 19 of each cycle.

\section{Blood Sampling for Pharmacokinetic Evaluation}

In 13 patients, blood samples were collected at Hours $0,2,2.25,2.5,2.75,3.0,3.5,4.0,6.0,8.0,10.0$, $12.0,14.0,16.0,24.0,26.0$, and 28.0 during the first ifosfamide infusion of the first cycle. Additional patients had blood samples taken at Hours 0, 14, and 26 of ifosfamide infusion. With each sample, a total of $6 \mathrm{~mL}$ of blood was collected, centrifuged, separated into plasma, and frozen at $-20^{\circ} \mathrm{C}$. Ifosfamide, 2-DCE, and 3-DCE were measured via a gas chromatograph/mass spectrometer (GC/MS)-stable isotope method. The accuracy and precision of ifosfamide quantitation is $107.5 \%$ and $4.7 \%$, respectively, at 1.5 $\mu \mathrm{mol} / \mathrm{L} ; 95.4 \%$ and $1.37 \%$, respectively, at $20.0 \mu \mathrm{mol} /$ $\mathrm{L}$; and $95.4 \%$ and $1.37 \%$, respectively, at $100 \mu \mathrm{mol} / \mathrm{L}$.

Urine was collected for 24 hours after the start of the first ifosfamide infusion in a random subset of patients treated on trial. Plasma and urine samples were spiked with an internal standard solution containing the oxazaphosphorine derivative trofosfamide and were subsequently extracted twice with dichloromethane. Simultaneous determination of ifosfamide, its dechloroethyl metabolites, and trofosfamide was performed by means of nitrogen/phosphorus (N/P) flame ionization gas chromatography/nitrogen phosphorus flame ionization (GC/NPFID) using a fusedsilica capillary column (HP5 [5\% PhMe Silicone]) under isothermal conditions, with $\mathrm{N}_{2}$ serving as the carrier gas. The temperature of the oven, injector, and detector were set at $195^{\circ} \mathrm{C}, 300^{\circ} \mathrm{C}$, and $300^{\circ} \mathrm{C}$, respectively. ${ }^{18}$

\section{Erythromycin Breath Test}

Four $\mu \mathrm{Ci}$ of $\left[{ }^{14} \mathrm{C} \mathrm{N}\right.$-methyl] erythromycin dissolved in $2 \mathrm{~mL}$ of normal saline was injected intravenously into patients before the initiation of any therapy. ${ }^{14} \mathrm{~A}$ single sample of exhaled carbon dioxide was taken 20 minutes later into vials that contained $4 \mathrm{~mL}$ of benzethonium hydroxide and ethanol $(1: 1)$ and a trace amount of thymopthalein. A total of $12 \mathrm{~mL}$ of Aquasol was added to each sample, which was then sealed in darkness for 24 hours. A scintillation counter measured the amount of radioactivity present. The percentage of administered carbon exhaled per minute was calculated assuming an endogenous carbon dioxide production of $5 \mathrm{mmol} \mathrm{CO}_{2} / \mathrm{m}^{2} / \mathrm{min}$.

\section{Toxicity Measurements}

National Cancer Institute (NCI) Common Toxicity Criteria (version 2.0) were used to classify adverse events.

\section{Statistical Analysis}

The area under the curve (AUC) was calculated for the time period 0 to 24 hours using the standard trapezoidal method. For ifosfamide only, Kel, the elimination rate constant, was calculated using a mixed effects model for the $\log 10$ of ifosfamide concentration. Each patient was allowed to have a random intercept (3-hour plasma measurement). The Kel values were used to complete the exponential decay of ifosfamide to calculate the AUC(0 to infinity). Metabolite AUC and 14-hour levels, and ERMBT and 14-hour drug levels were compared using Spearman correlation coefficient. The occurrence of toxicity was dichotomized by grade into serious (grades 3 and 4) and none to mild/moderate (grades 0 to 2) using the NCI Common Toxicity Criteria. Comparisons between continuous pharmacokinetic parameters and dichotomous toxicity outcomes were conducted using the Wilcoxon rank-sum test statistic, which tests whether the median values between toxicity groups were similar.

The pattern of changes in hematological parameters (white blood cell count [WBC], platelets, hemoglobin) over the first 4 cycles was analyzed using a random effects model including fixed effects for dose, cycle, day within each cycle, and interactions. Measurements of serum creatinine were converted into creatinine clearance using the Cockcroft-Gault formula.

\section{RESULTS \\ Ifosfamide, 2-DCE, 3-DCE Level at 14 Hours Correlates With AUC}

Thirteen patients had extensive sampling of ifosfamide, 2-DCE, and 3-DCE levels after the first dose of ifosfamide, which was used to calculate an $\operatorname{AUC}\left(0_{-}\right.$ 
TABLE 1

Correlation of Drug AUC to 14-Hour Level in 13 Patients

\begin{tabular}{llll}
\hline Metabolite & AUC & Correlation coefficient* & $\boldsymbol{P}$ \\
\hline Ifosfamide & $0-24$ & 0.97 & $<.0001$ \\
Ifosfamide & 0 -infinity & 0.97 & $<.0001$ \\
2-DCE & $0-24$ & 0.94 & $<.0001$ \\
3-DCE & $0-24$ & 0.95 & $<.0001$ \\
\hline
\end{tabular}

AUC indicates area under the curve; 2-DCE, 2-dichloroethylifosfamide; 3-DCE, 3-dichloroethylifosfamide.

* Spearman correlation coefficient

$24 \mathrm{~h})$. The coefficients of elimination of ifosfamide used to calculate the AUC( 0 to infinity) were 0.04398 and 0.03889 for the high- and low-dose groups, respectively.

The AUC $(n=13)$ values for each metabolite were found to be strongly correlated with their 14hour (peak) levels (Table 1). Given this robust relation and the difficulty in obtaining extensive sampling of these metabolites in all patients, we collected 14-hour drug levels in 41 additional patients and performed further correlations based on these values. This provides the basis for future evaluations of ERMBT and toxicity using our larger sample size of 14 -hour metabolite levels $(n=54)$.

\section{ERMBT Results Do Not Correlate With Ifosfamide 14-Hour Levels}

ERMBT was performed on 81 patients. Thirteen patients had ifosfamide and metabolite AUC data for comparison and 52 patients had 14-hour levels available for comparison. There was no relation observed between ifosfamide, 2-DCE, 3-DCE AUC or 14-hour levels and ERMBT (Table 2).

\section{Urinary Ifosfamide and Metabolites Concentration Correlates With 14-Hour Plasma Levels}

Twenty-five patients treated on the high-dose ifosfamide arm had 24-hour urinary metabolite evaluations. The median total amount (in micromoles) excreted for ifosfamide, 2-DCE, and 3-DCE were 2377 (range, 1075-4416), 814 (range, 288-1450), and 893 (range, 455-1703), respectively. Twenty-nine patients treated with standard dose ifosfamide had median total amounts (in micromoles) of ifosfamide, 2-DCE, and 3-DCE excreted in the urine of 3680 (range, 7058622), 1101 (range, 301-3253), and 1500 (range, 3632974). The 14-hour plasma measurements were positively and significantly correlated with the 24-hour urinary concentration (Table 3 ).
TABLE 2

Correlation Between ERMBT and Ifosfamide and Metabolites

\begin{tabular}{lllll}
\hline Sample size & Metabolite & Measurement & Correlation coefficient* & $\boldsymbol{P}$ \\
\hline 13 & Ifosfamide & $\mathrm{AUC}_{0-24}$ & 0.016 & .96 \\
13 & Ifosfamide & $\mathrm{AUC}_{0 \text {-infinity }}$ & 0.016 & .96 \\
13 & 2-DCE & $\mathrm{AUC}_{0-24}$ & 0.14 & .65 \\
13 & 3-DCE & $\mathrm{AUC}_{-24}$ & 0.17 & .59 \\
52 & Ifosfamide & 14 -hour & -0.34 & .087 \\
52 & 2-DCE & 14-hour & -0.13 & .36 \\
52 & 3-DCE & 14-hour & -0.13 & .35 \\
\hline
\end{tabular}

ERMBT indicates erythromycin breath testing; AUC, area under the curve; 2-DCE, 2-dichloroethylifosfamide; 3-DCE, 3-dichloroethylifosfamide.

* Spearman correlation coefficient.

\section{Characterization of Hematologic Toxicity in Patients Treated on High- vs Low-Dose Ifosfamide Arms}

WBCs, ANCs, hemoglobin, and platelet counts were determined at 7 different time points during each cycle of therapy. This information is plotted in Figure 2 and reveals the differential degree of hematologic toxicity in patients treated on high-dose or low-dose ifosfamide arms in conjunction with granulocyte-colony stimulating factor (GCSF). Leukopenia, anemia, and thrombocytopenia were found to be more severe in the high-dose ifosfamide arm and worsened with subsequent cycles of chemotherapy.

Patients on the low-dose ifosfamide arm exhibited 2 WBC peaks each cycle. This potentially indicates an early rise in WBC due to GCSF therapy followed by suppression due to chemotherapy and a second peak due to continued GCSF effect. The decrement in peak observed in the high-dose arm during the fourth cycle of chemotherapy may reflect depletion of stem cells.

Reduction in hemoglobin was cumulative and worsened with each subsequent cycle in both doses. Platelet counts dropped more dramatically in the high-dose arm and worsened with each cycle.

\section{Correlation of Hematologic Toxicity With 14-Hour Levels of Ifosfamide and Metabolites, ERMBT, and 24-Hour Urinary Concentrations}

Fourteen-hour levels of ifosfamide, 2-DCE, and 3DCE were compared with hematologic toxicity experienced during the first cycle of treatment. There was a statistically significant difference in the 14hour levels of each metabolite and both neutropenia and thrombocytopenia (Table 4). Each $10-\mu \mathrm{L} / \mathrm{L}$ incremental increase in the 14-hour ifosfamide level increased the likelihood of grade $3 / 4$ neutropenia by more than 2 -fold (odds ratio of $2.1 ; 95 \%$ confidence interval, 1.4-3.1). No correlation was observed with 
TABLE 3

Correlation Between 14-Hour Plasma Levels and 24-Hour Urine Concentration of Ifosfamide and Metabolites

\begin{tabular}{llll}
\hline & \multicolumn{3}{c}{ 24-hour urine concentration $(\mu \mathrm{mol} / \mathrm{L})$} \\
\cline { 2 - 4 } 14-hour metabolite plasma level & Ifosfamide & 2-DCE & 3-DCE \\
\hline Ifosfamide: correlation coefficient/sample size & $0.62(P<.0001) / 51$ & $0.50(P=.0003) / 50$ & $0.59(P<.0001) / 51$ \\
2-DCE: correlation coefficient/sample size & $0.60(P<.0001) / 51$ & $0.64(P<.0001) / 50$ & $0.70(P<.0001) / 51$ \\
3-DCE: correlation coefficient/sample size & $0.49(P=.0003) / 51$ & $0.54(P<.0001) / 50$ & $0.68(P<.0001) / 51$ \\
\hline 2-DCE indicates 2-dichloroethylifosfamide; 3-DCE, 3-dichloroethylifosfamide. & & \\
\hline
\end{tabular}

hemoglobin toxicity, except with 3 -DCE, but only 4 patients were found to experience grade 3/4 toxicity after 1 cycle of therapy.

ERMBT results were also compared with hematologic toxicity and controlled for patients treated on the high-dose and standard-dose ifosfamide arm. No correlation was noted with ERMBT results and ANC and hemoglobin toxicity. Patients with an ERMBT $\leq 2.0$ were more likely to experience severe thrombocytopenia.

Urinary concentrations of metabolites were found to exhibit a statistically significant correlation with ANC toxicity (Table 5), but there was no significant association noted with either hemoglobin or platelet toxicity.

\section{Correlation of Nonhematologic Toxicity With 14-Hour Levels of Ifosfamide and Metabolites and ERMBT}

In all, 52 patients with relevant pharmacokinetic data and creatinine toxicity data were evaluated. Two patients experienced grade 1 toxicity, 2 patients experienced grade 2 toxicity, and 1 patient experienced grade 4 toxicity. Despite the small numbers of patients experiencing renal toxicity, there was a significant difference in 14-hour plasma levels in patients with grade $0 / 1$ toxicity compared with grade $2 / 3 / 4$ toxicity (Table 6 ). Median levels of ifosfamide, 2-DCE, and 3-DCE in patients experiencing grade 2/ $3 / 4$ creatinine toxicity were more than twice that of other patients. No correlation was identified with 24hour urinary concentrations or ERMBT results after stratifying for both high-dose and low-dose ifosfamide arms (data not shown).

Seven patients with available pharmacokinetic data were found to have any form of neurologic toxicity. No statistically significant difference was noted in the 14-hour plasma levels and ERMBT results when comparing patients with and without neurologic toxicity. However, the trend was similar to that observed with renal toxicity. Patients with grade 0 neurologic toxicity were found to have a statistically significant difference in 24-hour urinary concentra- tion values compared with patients with grade 1-3 toxicities (498.6 vs $805.6 ; P=.019$ ). No other correlations were observed with the urinary concentrations.

\section{DISCUSSION}

In the current study, we described ifosfamide pharmacokinetics in a trial of soft-tissue sarcoma patients treated with $6 \mathrm{~g} / \mathrm{m}^{2}$ or $12 \mathrm{~g} / \mathrm{m}^{2}$ of ifosfamide in combination with doxorubicin $\left(60 \mathrm{mg} / \mathrm{m}^{2}\right)$. We demonstrated that the 14-hour level of ifosfamide taken on Day 1 of a 3-day treatment is a useful substitute in describing the pharmacokinetics of ifosfamide, and correlates well with hematologic toxicity in the first cycle of therapy. Clinically, this observation may be useful in treating patients at high risk for complications with myelosuppression (ie, underlying lowgrade infections, poor performance status). With further validation, it could potentially allow for decreasing the dose of ifosfamide on subsequent treatment days to decrease the risks associated with hematologic toxicity. Of course, such an intervention would also decrease the tumor's dose of ifosfamide, which may or may not impact patient outcomes. ${ }^{17}$

As hematologic toxicities progressively worsen in subsequent cycles (Fig. 2), the value of this observation made only during the first cycle of treatment could be questioned. In addition, ifosfamide has been reported to be subject to autoinduction and thus drug metabolism may increase over time. ${ }^{3,19,20}$ Evaluation of similar pharmacokinetic parameters in subsequent cycles may provide useful information.

Renal and neurologic toxicities were reported to occur relatively infrequently in this limited population, making it difficult to reach conclusions regarding the predictive value of pharmacokinetic parameters. A significant difference was observed for all metabolites and renal toxicity. A significant difference was noted only in 14-hour plasma levels of ifosfamide in patients with and without any neurologic toxicity. Because chloroacetaldehyde is believed to be the metabolite responsible for neurologic toxicity, we 

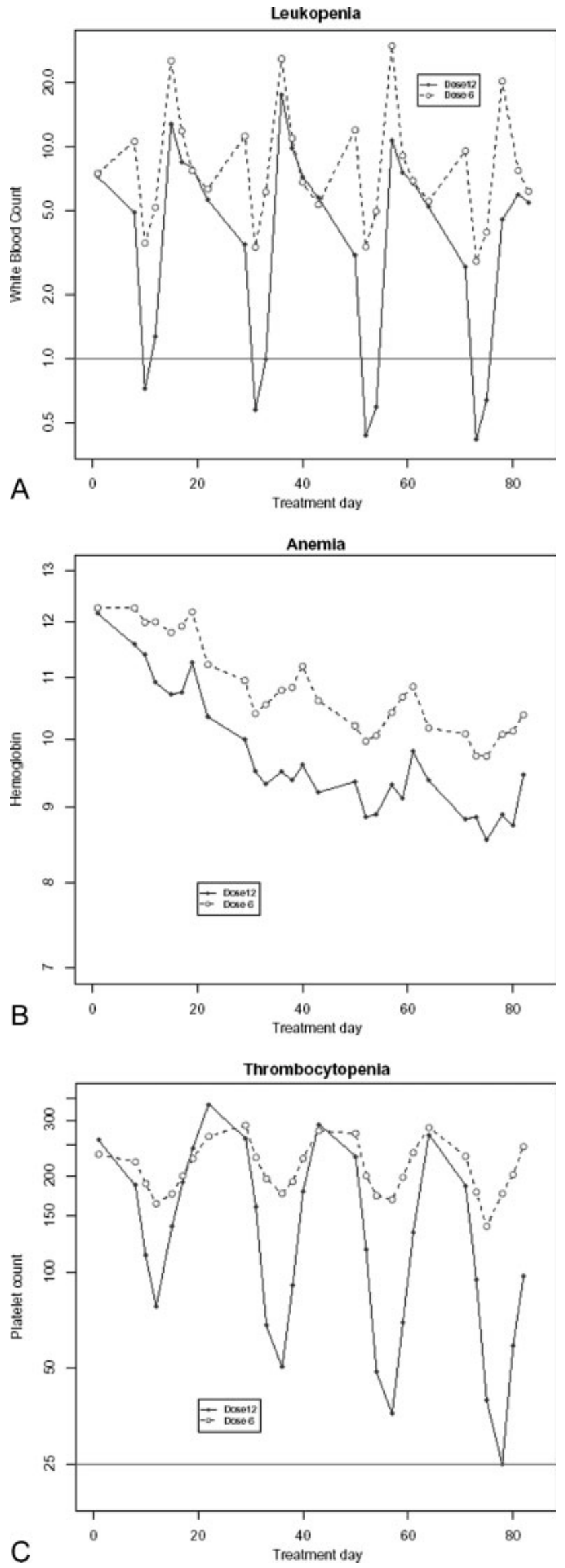

TABLE 4

Hematologic Toxicity and Corresponding Median Plasma Levels of Ifosfamide and Metabolites and ERMBT Values

\begin{tabular}{|c|c|c|c|c|c|}
\hline \multirow[b]{2}{*}{ Toxicity/Grade* } & \multicolumn{3}{|c|}{ 14-hour plasma levels $(\mu \mathrm{mol} / \mathrm{L})$} & \multicolumn{2}{|c|}{$\begin{array}{l}\text { No. of patients (\%) } \\
\text { with ERMBT }\end{array}$} \\
\hline & Ifosfamide & 2-DCE & 3-DCE & $\leq \mathbf{2 . 0}$ & $>2.0$ \\
\hline \multicolumn{6}{|l|}{ ANC } \\
\hline $0-2(\mathrm{n}=24)$ & 64.6 & 12.0 & 13.9 & $8(47.1)$ & $16(45.7)$ \\
\hline $3-4(n=28)$ & 123.5 & 19.7 & 26.8 & $9(52.9)$ & $19(54.3)$ \\
\hline$P^{\dagger}$ & $<.0001$ & .0015 & .0016 & $P^{\ddagger}$ & .9999 \\
\hline \multicolumn{6}{|l|}{ Platelets } \\
\hline $0-2(\mathrm{n}=45)$ & 80.8 & 16.0 & 17.9 & $12(70.6)$ & $33(94.3)$ \\
\hline $3-4(n=7)$ & 155.0 & 30.9 & 39.6 & $5(29.4)$ & $2(5.7)$ \\
\hline$P$ & .0016 & .014 & .0086 & $p^{\ddagger}$ & .0309 \\
\hline \multicolumn{6}{|l|}{ Hemoglobin } \\
\hline $0-2(\mathrm{n}=48)$ & 91.5 & 16.1 & 21.7 & $15(88.2)$ & $33(94.3)$ \\
\hline $3-4(n=4)$ & 105.2 & 30.2 & 54.5 & $2(11.8)$ & $2(5.7)$ \\
\hline$P$ & .43 & .064 & .030 & $p^{\$}$ & .5890 \\
\hline
\end{tabular}

ERMBT indicates erythromycin breath testing; 2-DCE, 2-dichloroethylifosfamide; 3-DCE, 3-dichloroethylifosfamide; ANC, absolute neutrophil count.

* Toxicity was graded according to the National Cancer Institute's Common Toxicity Criteria (version 2.0).

$\dagger$ Wilcoxon rank-sum test.

\$ Fisher exact test.

TABLE 5

Hematologic Toxicity and Corresponding Median 24-Hour Urinary Concentrations

\begin{tabular}{llll}
\hline & \multicolumn{3}{c}{ 24-hour urinary concentration $(\mu \mathrm{mol} / \mathrm{L})$} \\
\cline { 2 - 4 } Toxicity/Grade* & Ifosfamide & 2-DCE & 3-DCE \\
\hline ANC & & & \\
$0-2(\mathrm{n}=23)$ & 409.9 & 148.9 & 151.0 \\
$3-4(\mathrm{n}=28)$ & 627.7 & 227.1 & 283.6 \\
$P^{\dagger}$ & .0051 & .0246 & .0020 \\
Platelets & & & \\
$0-2(\mathrm{n}=44)$ & 498.6 & 174.5 & 214.3 \\
$3-4(\mathrm{n}=7)$ & 610.8 & 192.2 & 272.9 \\
$P$ & .13 & .53 & .23 \\
Hemoglobin & & & \\
$0-2(\mathrm{n}=47)$ & 530.3 & 187.7 & 235.4 \\
$3-4(\mathrm{n}=4)$ & 555.9 & 168.4 & 273.3 \\
$P$ & .65 & .97 & .55 \\
\hline
\end{tabular}

2-DCE indicates 2-dichloroethylifosfamide; 3-DCE, 3-dichloroethylifosfamide; ANC, absolute neutrophil count.

* Toxicity was graded according to the National Cancer Institute's Common Toxicity Criteria (version 2.0).

$\dagger$ The Wilcoxon rank-sum test was used to test the equality of the median between toxicity groups.

FIGURE 2. Hematologic toxicity. This figure demonstrates the predicted average values of leukopenia, anemia, and thrombocytopenia for the first 4 treatment cycles. Results are derived from fitting a random effects model to all data. 
TABLE 6

Nonhematologic Toxicity and Corresponding Plasma Levels of Ifosfamide and Metabolites

\begin{tabular}{llll}
\hline & \multicolumn{3}{c}{ 14-hour plasma levels } \\
\cline { 2 - 4 } Toxicity/Grade* & Ifosfamide & 2-DCE & 3-DCE \\
\hline Creatinine & & & \\
$0-1(\mathrm{n}=49)$ & 83.1 & 16.1 & 21.5 \\
$2-4(\mathrm{n}=3)$ & 185.7 & 39.2 & 51.7 \\
$P^{\dagger}$ & .010 & .0081 & .0091 \\
Neurologic & & & \\
$0(\mathrm{n}=45)$ & 83.1 & 16.1 & 21.9 \\
$1-3(\mathrm{n}=7)$ & 141.0 & 29.9 & 34.9 \\
$P$ & .063 & .23 & .28 \\
\hline
\end{tabular}

2-DCE indicates 2-dichloroethylifosfamide; 3-DCE, 3-dichloroethylifosfamide.

* Toxicity was graded according to the National Cancer Institute's Common Toxicity Criteria (version 2.0).

$\dagger$ The Wilcoxon rank-sum test was used to test the equality of the median between toxicity groups.

expected to see an increase in both 2-DCE and 3DCE metabolites. Given the low incidence of these toxicities in this trial, it is not surprising that only a trend and not a more definitive association was observed.

Although drug and metabolite urine concentrations and total amount excreted were found to be significantly correlated with pharmacokinetic parameters, the correlation was not robust. Collecting 24hour urine samples even in a controlled setting is problematic, and the practical value of urine sampling clinically for drug toxicity prediction is likely limited.

ERMBT was not found to be of value in predicting ifosfamide pharmacokinetics or toxicity in this study of combination ifosfamide and doxorubicin therapy. Although the correlation between lower ERMBT value and more severe thrombocytopenia was considered significant, there were relatively few numbers of affected patients.

ERMBT has been shown to be a reproducible assessor of hepatic CYP3A4 activity in previous studies and has shown to predict the clearance of CYP3A4 substrates, including docetaxel and cyclosporine. ${ }^{15,21}$ Although CYP3A4 is identified as the main enzyme involved in ifosfamide activation and metabolism, CYP3A5 and CYP2B6 have also been shown to be involved to some extent, and are not measured by the ERMBT (Fig. 1). ${ }^{5-7,15,22}$

In addition, although ERMBT has been shown to correlate with CYP3A activity, the correlation between ifosfamide and CYP3A levels is complex. CYP3A is mainly responsible for the transformation of ifosfamide to active product and the metabolism to inactive product. Additional work to separate out these 2 processes may be necessary to arrive at a predictive pharmacokinetic test. The concomitant use of dexamethasone and other CYP3A4 inducers and inhibitors by study patients may or may not serve as additional confounding factors. ${ }^{16,22,23}$ The CYP3A family may also play a role in doxorubicin metabolism, which was coadministered with ifosfamide in this trial. ${ }^{24}$

The ERMBT has been the subject of much pharmacologic criticism. Full discussion has been reviewed elsewhere. ${ }^{25}$ To summarize, ERMBT has to date been shown to be a reasonable predictor of CYP3A activity of some, but not all, substrates. Many additional probes exist for evaluating CYP3A activity including midazolam, dextromethorphan, verapamil, and cortisol. ${ }^{26}$ The midazolam clearance test is currently the subject of investigation and offers the theoretical advantage of assessing both hepatic and intestinal CYP3A4 and CYP3A5. ${ }^{27}$ It is interesting to note that in some studies the ERMBT has been found to have a significantly positive correlation with midazolam clearance, whereas in others no correlation was observed. ${ }^{27,28}$ The midazolam clearance test has been shown to be more predictive than the ERMBT in a study examining the pharmacokinetics of the topoisomerase I inhibitor irinotecan, another antineoplastic agent with complex metabolism involving both CYP3A4 and uridine diphosphate glucoronosyltransferase $1 \mathrm{Al}^{29}$ Therefore, because the ERMBT did not appear to be useful in this setting, testing using other probes in future studies may prove to be more fruitful.

There may be additional factors that may be useful in predicting ifosfamide toxicity that were not evaluated in the current study. Ifosfamide is administered as a racemic mixture, and some studies suggest that $(\mathrm{R})$ ifosfamide may have a more favorable efficacy and toxicity profile. ${ }^{30}$ There also may be enantioselective differences in ifosfamide metabolism. Specifically, in vitro studies have shown that CYP2B6 may be the key enzyme in creating (S)-2-DCE and (S)-3-DCE. ${ }^{7}$ We did not investigate the difference in stereoisomers of ifosfamide in this clinical investigation or any in vivo probes of CYP2B6 activity.

Although the metabolism of ifosfamide has been studied extensively, it remains poorly understood. In the current study, we demonstrated that pharmacokinetic data correlate with some forms of ifosfamide toxicity and therefore have the potential to affect clinical practice. Further validation of the use of pharmacokinetic parameters in a prospective setting may prove valuable. Although ERMBT was not found to be a useful in vivo probe for our patients treated with ifosfamide and doxorubicin, further investigation of in vivo CYP3A4 activity using the midazolam 
clearance test or other in vivo probes may prove to be more beneficial.

\section{REFERENCES}

1. Zalupski M, Baker LH. Ifosfamide. J Natl Cancer Inst. 1988;80:556-566.

2. Van Dyk JJ, Falkson HC, Van der Merwe AM, et al. Unexpected toxicity in patients treated with iphosphamide. Cancer Res. 1972;32:921-924.

3. Kerbusch T, de Kraker J, Keizer HJ, et al. Clinical pharmacokinetics and pharmacodynamics of ifosfamide and its metabolites. Clin Pharmacokinet. 2001;40:41-62.

4. McCune JS, Risler LJ, Phillips BR, et al. Contribution of CYP3A5 to hepatic and renal ifosfamide N-dechloroethylation. Drug Metab Dispos. 2005;33:1074-1081.

5. Walker D, Flinois JP, Monkman SC, et al. Identification of the major human hepatic cytochrome P450 involved in activation and $\mathrm{N}$-dechloroethylation of ifosfamide. Biochem Pharmacol. 1994;47:1157-1163.

6. Huang Z, Roy P, Waxman DJ. Role of human liver microsomal CYP3A4 and CYP2B6 in catalyzing N-dechloroethylation of cyclophosphamide and ifosfamide. Biochem Pharmacol. 2000;59:961-972.

7. Granvil CP, Madan A, Sharkawi M, et al. Role of CYP2B6 and CYP3A4 in the in vitro $\mathrm{N}$-dechloroethylation of (R)and (S)-ifosfamide in human liver microsomes. Drug Metab Dispos. 1999;27:533-541.

8. Sladek NE. Metabolism of cyclophosphamide by rat hepatic microsomes. Cancer Res. 1971;31:901-908.

9. Sladek NE. Metabolism of oxazaphosphorines. Pharmacol Ther. 1988;37:301-355.

10. Brock N, Hilgard P, Peukert M, et al. Basis and new developments in the field of oxazaphosphorines. Cancer Invest. 1988;6:513-532.

11. Goren MP, Wright RK, Pratt CB, et al. Dechloroethylation of ifosfamide and neurotoxicity. Lancet. 1986;2:1219-1220.

12. Ruzicka JA, Ruenitz PC. Cytochrome P-450-mediated Ndechloroethylation of cyclophosphamide and ifosfamide in the rat. Drug Metab Dispos. 1992;20:770-772.

13. Kerbusch T, Mathjt RA, Keizer HJ, et al. Population pharmacokinetics and exploratory pharmacodynamics of ifosfamide and metabolites after a 72-h continuous infusion in patients with soft tissue sarcoma. Eur J Clin Pharmacol. 2001;57:467-477.

14. Watkins PB, Murray SA, Winkelman LG, et al. Erythromycin breath test as an assay of glucocorticoid-inducible liver cytochromes P-450. Studies in rats and patients. J Clin Invest. 1989;83:688-697.

15. Watkins PB. Noninvasive tests of CYP3A enzymes. Pharmacogenetics. 1994;4:171-184.

16. Hirth J, Watkins PB, Strawderman M, et al. The effect of an individual's cytochrome CYP3A4 activity on docetaxel clearance. Clin Cancer Res. 2000;6:1255-1258.
17. Worden FP, Taylor JM, Biermann JS, et al. Randomized phase II evaluation of $6 \mathrm{~g} / \mathrm{m} 2$ of ifosfamide plus doxorubicin and granulocyte colony-stimulating factor (G-CSF) compared with $12 \mathrm{~g} / \mathrm{m} 2$ of ifosfamide plus doxorubicin and G-CSF in the treatment of poor-prognosis soft tissue sarcoma. J Clin Oncol. 2005;23:105-112.

18. Kurowski V, Wagner T. Comparative pharmacokinetics of ifosfamide, 4hydroxyifosfamide, chloroacetaldehyde, and 2and 3-dechloroethylifosfamide in patients on fractionated intravenous ifosfamide therapy. Cancer Chemother Pharmacol. 1993;33:36-42.

19. Boddy AV, Cole M, Pearson AD, et al. The kinetics of the auto-induction of ifosfamide metabolism during continuous infusion. Cancer Chemother Pharmacol. 1995;36:53-60.

20. Kerbusch T, Huitema AD, Ouwerkerk J, et al. Evaluation of the autoinduction of ifosfamide metabolism by a population pharmacokinetic approach using NONMEM. Br J Clin Pharmacol. 2000;49:555-561.

21. Watkins PB, Hamilton TA, Annesley TM, et al. The erythromycin breath test as a predictor of cyclosporine blood levels. Clin Pharmacol Ther. 1990;48:120-129.

22. McCune JS, Hawke RL, LeCluyse EL, et al. In vivo and in vitro induction of human cytochrome P4503A4 by dexamethasone. Clin Pharmacol Ther. 2000;68:356-366.

23. Singer JM, Hartley JM, Brennan C, et al. The pharmacokinetics and metabolism of ifosfamide during bolus and infusional administration: a randomized cross-over study. Br J Cancer. 1998;77:978-984.

24. Kivisto KT, Kroemer HK, Eichelbaum M. The role of human cytochrome P450 enzymes in the metabolism of anticancer agents: implications for drug interactions. Br J Clin Pharmacol. 1995;40:523-530.

25. Chiou WL, Jeong HY, Wu TC, et al. Use of the erythromycin breath test for in vivo assessments of cytochrome P4503A activity and dosage individualization. Clin Pharmacol Ther. 2001;70:305-310.

26. Krecic-Shepard ME, Barnas CR, Slimko J, et al. In vivo comparison of putative probes of CYP3A4/5 activity: erythromycin, dextromethorphan, and verapamil. Clin Pharmacol Ther. 1999;66:40-50.

27. Kinirons MT, O'Shea D, Kim RB, et al. Failure of erythromycin breath test to correlate with midazolam clearance as a probe of cytochrome P4503A. Clin Pharmacol Ther. 1999; 66:224-231.

28. Lown KS, Thummel KE, Benedict PE, et al. The erythromycin breath test predicts the clearance of midazolam. Clin Pharmacol Ther. 1995;57:16-24.

29. Mathijssen RH, de Jong FA, van Schaik RH, et al. Prediction of irinotecan pharmacokinetics by use of cytochrome P450 3A4 phenotyping probes. J Natl Cancer Inst. 2004;96:1585-1592.

30. Roy P, Tretyakov O, Wright J, et al. Stereoselective metabolism of ifosfamide by human P-450s 3A4 and 2B6. Favorable metabolic properties of R-enantiomer. Drug Metab Dispos. 1999;27:1309-1318. 